\title{
Complexity of Converter Placement Supporting Broadcast in WDM Networks
}

\author{
Rudra Dutta, Prashant Iyer and Carla D. Savage \\ Computer Science department, North Carolina State University \\ Raleigh, NC
}

\begin{abstract}
Wavelength converters simplify the wavelength assignment problem in virtual topology design in optical networks and increase the utilization of the fiber bandwidth. However, converters are costly, and minimizing the number of converters needed to support a given level of functionality has been investigated in the literature in various contexts. In particular, previous work has addressed the problem of minimizing the number of converters needed to support broadcast over all network nodes with a given set of residual wavelengths on each link. In this paper, we show that previous work leaves the computational complexity of this question open. We go on to show that the problem is in fact NP-Complete, but becomes tractable in the special case when the network graph is a tree. We also show that there are cases when the heuristics articulated in previous work fail to provide solutions, and provide some heuristic approaches to solve the general problem.
\end{abstract}

Keywords: Optical networks, network provisioning, wavelength converter, broadcast, WDM, NP-Completeness, graph coloring

\section{INTRODUCTION}

\section{A. General Context}

In the last decade or so, fiber-optic communication has brought a revolution in the area of wide area networking. Optical networks are widely regarded as the solution of choice for the high performance networks that will form the national backbones of tomorrow, as well as networks lower in the planetary network hierarchy. In particular, the high performance and high predictability achievable using optical networking has made it the technology of choice in many telecommunication backbones. This revolution was initially fueled by the highly desirable characteristics of fiber-optic communication, namely very high bandwidth, very low bit error rates, and very low fiber cost. Wavelength Division Multiplexing (WDM), the use of multiple wavelength channels over a single fiber carrying data independently, multiplied the available bandwidth to still higher values. Wavelength channels currently carry 10 or $40 \mathrm{Gbps}$, and commercial implementations with 100 or 200 wavelength channels will soon enter the market, leading to Tbps capability in a single fiber.

In a wide area network, most node pairs cannot be connected to each other by a physical fiber link but must reach each other using other nodes as intermediaries. Data traffic generated by one node thus generally follows a multi-hop path through the network, with intermediate nodes forwarding the traffic

This research was supported in part by NSF grant \# ANI-0322107. appropriately according to some routing policy. Network nodes thus act as routers or switches for traffic generated by other nodes. The Tbps data rates enabled by optical communication and WDM are several orders of magnitude beyond the capability of current data switches or routers. This is because such routers are electronic devices with sophisticated computing ability, and usually complicated routing decisions to perform for each unit of data traffic, such as a packet. Thus the data rates achievable over optical networks would be limited to many times less than the capacity of the fibers due to the forwarding bottleneck, if purely electronic switches were to be used exclusively. Fortunately, advances in optical device technology has made it possible to go past this bottleneck by switching a large part of the traffic in the optical domain itself. This allows the construction of Wavelength Switches, which have commonly come to be known as Optical CrossConnects (OXC). In such an OXC, entire wavelenth channels from an incoming fiber link can be switched to an outgoing fiber link without intermediate conversion to electronic data (the so-called Opto-Electro-Optic or OEO conversion). Only wavelength channels which contain traffic that has to be delivered at this node, or in which traffic is to be injected by this node, has to be terminated from the optical to the electronic domain, and switched by a traditional electronic router. Such an electronic router is called a Digital CrossConnect (DXC) in this context. Thus clear optical channels or lightpaths are created over multiple physical hops. The problem of determining the set of such lightpaths to be formed in the network to allow maximum wavelength utilization (or minimizing delay for traffic demand components, or other objectives) is generally called the virtual topology design problem. An instance of a class of virtual topology design problems is the traffic grooming problem, where the objective is to minimize the OEO conversion in the network. The literature on virtual topology design and traffic grooming is extensive, and has been surveyed in [6], [7].

The limitation of lightpath formation is the wavelength continuity constraint: after optical switching, the wavelength of the outgoing optical channel is the same as the incoming one. Thus a lightpath cannot be formed if the target wavelength is not free on the intended outgoing link. This may require a lightpath to be terminated even when no traffic inside it is required to be terminated. Such is the case when enabling broadcast communication; i.e. the transfer of traffic originated by one node to every node in the network. The limitation of 
wavelength continuity can be overcome by using wavelength converters, devices that translate the wavelength of a channel entirely in the optical domain. Thus a broader range of virtual topology solutions can be implemented without sacrificing the benefits of optical switching. However, wavelength converters are expensive, unlike the simple wavelength-continuous optical switches, and this is likely to remain true in the foreseeable future.

\section{B. Prior Work}

Because of the above considerations, there has been a significant amount of recent research in the area of efficient deployment of wavelength converters in a network [1]-[5], [9], [10], [13]-[15], [18], [20]-[28]. Most work has focused on a problem scenario with a given set of traffic demands to be carried. Generally traffic demands are assumed to exist between any pair of nodes, i.e. traffic is all-to-all. The objective of design is to find the strategic placement of the minimum number of wavelength converters in the network to carry the given traffic. A variation of this problem is to find the minimum number of converters, and placements for them, such that any set of traffic demands which could be carried by a network with a wavelength converter at every node can still be carried. Studies have also addressed the issue of placing converters capable of partial wavelength conversion, which may be expected to be less costly than full converters.

A specific problem in this area is to place the minimum number of converters to enable all-optical broadcast. Broadcast is important in wide area networks because it is often required in control plane functioning of the network. This problem has been motivated and studied in [16], [17]. Since enabling broadcast usually comes after the primary concern of routing traffic demands, it is reasonable to assume that every fiber link has a specific set of residual wavelengths to support broadcast. No single wavelength can be expected to be free in all links of the network, thus wavelength converters are essential if all-optical broadcast is to be achieved. It is wellknown that efficient broadcast creates a spanning tree in the network; each node receives the data along one of the tree links and also forwards along every other link incident to it. This allows the broadcast to be achieved without any unnecessary replication of transmission. In the wavelength routed context, such a node does not need a wavelength converter if the data can be forwarded along each outgoing link on the same wavelength channel as it was received on. Thus the problem of converter placement supporting broadcast is posed in [16], [17] as the problem of finding an optically continuous spanning tree in a given network graph and assigning a wavelength to each link in the spanning tree from among the available wavelengths for that link, such that the number of nodes needing converters is minimized. Only full range converters are considered; i.e. a node with a converter can forward traffic using any available wavelength on each outgoing link, regardless of what wavelength the traffic was received on. We call this problem Broadcast Converter Node Selection (BCNS), following [17].
In both [16] and [17], the authors equate the BCNS problem with a problem we refer to as the Color-Connected Vertex Subset (CCVS) problem (see Section II-B). CCVS is proved to be NP-Complete as well as of bounded approximability in both [16] and [17], and heuristic solutions proposed. The approximability bound provided by the proof of [17] achieves an improvement over that of [16], and the heuristic approach is also a closer approximation than the older one.

\section{Our Contribution}

We have considered the same problem and realized that despite the substantial contributions of [16], [17], the complexity of BCNS remains unsettled because BCNS is not in fact equivalent to CCVS, contrary to the assertion of the authors of those earlier works. In this paper, we first show this inequivalence by providing instances where the two problems differ, and a solution to CCVS obtained by the algorithms provided in [16], [17] are not valid solutions of BCNS. We provide a precise formulation of the BCNS problem which allows us to clearly consider its complexity. Since all the NP-completeness related proofs of [16], [17] address the CCVS problem rather than the original BCNS problem, the complexity of the BCNS problem remains open. We settle this open question by showing that the problem is in fact polynomially solvable in tree networks, but can be very simply shown to be NP-Complete in general network graphs. The heuristic approaches provided in [16], [17] also address the CCVS problem, and on many instances will fail to provide feasible solutions to the BCNS problem. Then we provide some heuristic approaches and numerical results.

\section{PRoblem DEFINITION}

\section{A. The BCNS Problem}

The BCNS problem is defined precisely as follows. We are given an undirected connected network graph $G=(V, E)$. Wavelengths are indicated by integers in the range $1,2, \ldots W$, where $W$ is the number of distinct wavelengths supported by the physical transmission system used in the network. For each edge $e \in E$, a nonempty available color set $c(e) \subseteq$ $\{1 \ldots W\}$ is given. The problem is to choose a spanning tree $T=\left(V, E_{T}\right)$ of $G$, and a color $w(e) \in c(e)$ for each edge $e$ of $T$, such that the cardinality of the set of vertices $\left\{v \mid \exists e, e^{\prime} \in e_{T}(v): w(e) \neq w\left(e^{\prime}\right)\right\}$ is minimized, where $e_{T}(v)$ denotes the set of edges incident on the vertex $v$ in the spanning tree $T$.

We define some additional terminology to allow ease of discussion. For easy reference to [16], [17], we adopt some of the terminology of those earlier works, mapping them to our notations, which are slightly different. The set of available colors $c(e)$ is called the color-set of edge $e$. The set of edges incident on vertex $v$ in the given graph $G$ is denoted simply by $e(v)$. The vertex-color-set of a vertex $v$ is defined as the union of the color-sets of its incident edge; $v c(v)=\cup_{e \in e(v)} c(e)$. For each color $i$, the edge-set of $i$ is the set of all edges that have color $i$ available, $e c(i)=\{e \mid i \in c(e)\}$. 
In [16], [17], the authors generally assume that for each color $i$, the subgraph $G_{i}$ of $G$ consisting of only the edges $e c(i)$ and the vertices which form the endpoints of these edges is a connected subgraph. They correctly point out that if the subgraph is disconnected and consists of several connected components, it is straightforward to introduce fictitious extra colors and assign them to each connected component. Thus this assumption does not include any loss of generality. When constructing counterexamples, we stay within the bounds of this assumption; however, our own results do not require such an assumption.

Figure 1 shows an example. The highlighted vertices in the solution are equipped with converters, and hence can support different wavelength assignments in incident edges. It is easy

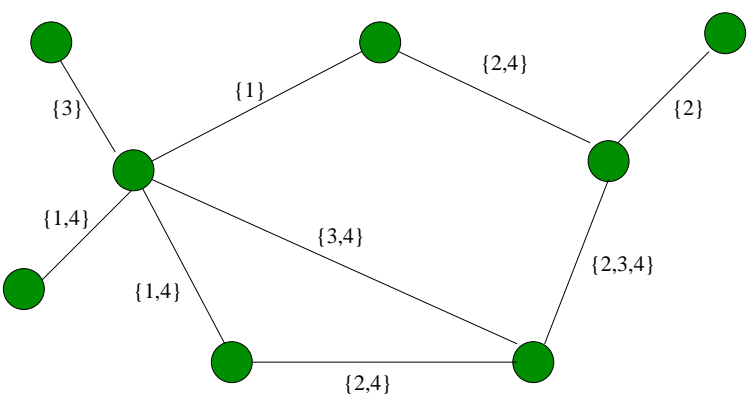

(a) A Network graph $G$, numbers on edges indicate $c(e)$

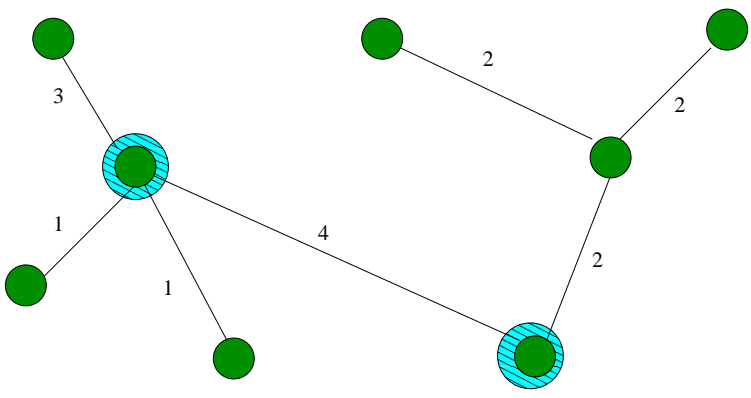

(b) A corresponding tree $T$,numbers on edges indicate $w(e)$

Fig. 1. An example of (a) an instance of BCNS and (b) a solution to the instance

to verify that this solution is indeed optimal, that is there is no solution using only one converter.

\section{B. The CCVS Problem}

The Color-Connected Vertex Subset problem is that of finding a minimum cardinality subset $S$ of vertices in the given network graph $G$ with the following two properties:

(i) If a graph $P(S)$ is constructed by starting with only the vertices $S$, and then connecting every pair of vertices $a$ and $b$ such that $v c(a)$ and $v c(b)$ have a non-empty intersection, $P(S)$ must form a connected graph. That is: $S$ must be colorconnected.

(ii) The set of all edges that have at least one available wavelength in common with the union of the available wavelengths on all incident edges to all vertices in $S$ must form a spanning subgraph of $G$. That is: the union of the vertex-color-sets of the vertices of $S$ must form a color-covering of $G$.

However, the set of vertices $S$ is not itself required to be connected by direct edges of $G$. This problem is thus one of finding a minimum cardinality color-connected subset of vertices such that the union of the vertex-color-sets of those vertices form a color-covering, in the nomenclature defined in [17].

\section{Relationship of BCNS and CCVS}

In the above example, the tree $T$ shown in $1(\mathrm{~b})$ is also a solution to the CCVS problem. This can be verified by noting that (i) the union of the vertex-color-sets of the two designated vertices is $\{1,2,3,4\}$, which is the set of all colors and thus certainly a color-covering, and (ii) the two vertices are colorconnected because the intersection of their vertex-color-sets is $\{1,3,4\} \cap\{2,3,4\}=\{3,4\}$ which is non-empty.

However, we have found that the requirements (i) and (ii), while obviously necessary, are not sufficient. In other words, a solution to CCVS on a graph $G$ is not necessarily a solution to BCNS on the same graph. Counterexamples are easy to construct. Figure 2 shows a graph $G_{1}$ that is such a counterexample. First, note that the components induced by

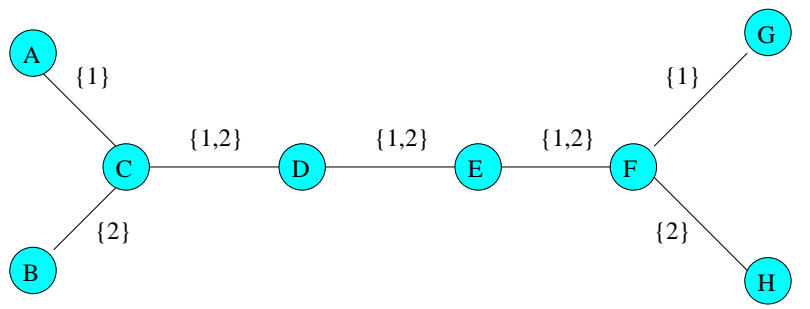

Fig. 2. A graph $G_{1}$ that is a counterexample to CCVS-BCNS equivalence

the edge-sets of color 1 and color 2 are each connected, so this graph meets the assumptions of [16], [17]. Consider the set of vertices $S_{1}=\{C, E\}$. The union of the vertex-colorsets of $C$ and $E$ is $\{1,2\}$, clearly a color-covering because all edges of $G$ include one or both colors in their color-sets. Also, the intersection of the vertex-color-sets of $C$ and $E$ is $\{1,2\}$, which is non-empty; thus $S_{1}$ is color-connected. Thus $S_{1}$ is a valid solution to the CCVS problem on graph $G_{1}$.

Now consider vertex $F$. It can reach vertices $G$ and $H$ using only color 1 and only color 2 respectively, thus it cannot reach both vertices unless it is equipped with a converter (irrespective of what color is used on the edge between vertices $E$ and $F$ ). Nodes $G$ and $H$ are connected to the rest of the graph only through vertex $F$, thus there cannot be a solution to BCNS on $G_{1}$ that does not put a converter at vertex $F$. Since $S_{1}$ does not include $F$, it cannot be a solution to BCNS on $G_{1}$. Therefore a solution to CCVS does not necessarily form a solution to BCNS.

\section{Complexity of BCNS}

Since CCVS and BCNS are not equivalent, the results regarding the computational complexity of CCVS that were 
obtained in [16], [17] leave the question of the complexity of the BCNS problem open. In this section we address this question. First, we show that BCNS can be proved to be NPComplete using results about the edge chromatic number of a graph. Then we go on to show that even though BCNS is NP-Complete in general, the problem becomes solvable in polynomial time when the graph $G$ is itself a tree. We also provide a straightforward algorithm that finds an optimal solution to tree instances of BCNS in polynomial time.

1) NP-Completeness of BCNS: A proper $k$-edge coloring of a graph $G$ is a function assigning one of the colors $\{1, \ldots, k\}$ to each edge of $G$ in such a way that edges incident with the same vertex are assigned different colors. The edge chromatic number of $G$, denoted $\chi(G)$, is the minumum number of colors, $k$ for which $G$ has a proper $k$-edge coloring. A well-known result due to Vizing is that either $\chi(G)=\Delta$ or $\chi(G)=\Delta+1$, where $\Delta$ is the maximum degree of any vertex in $G$ [29]. Although it is NP-complete in general graphs to decide whether $\chi(G)$ is $\Delta$ or $\Delta+1$ [11], there is a a polynomial time algorithm to produce a proper $\Delta+1$-edge coloring.

Theorem 2.1: BCNS is NP-Complete in general graphs. Proof: The decision version of BCNS is as follows. Given an instance comprised of a graph $G$, an integer $W$, a set $c(e) \subseteq$ $\{1, \ldots, W\}$ for each edge $e$, and an integer $q$ : is it possible to assign a wavelength $w(e) \in c(e)$ to each edge $e$ of a spanning tree $T$ of $G$ so that at most $q$ vertices of $G$ require converters, i.e., at most $q$ vertices have incident edges of $T$ that have been assigned different colors? Obviously, BCNS is in NP because a putative solution could be checked in time linear in the number of edges in the graph. We now show it to be NP-Complete by reducing it from Maximum Leaf Spanning Tree (MAXLEAF), which is known to be NP-Complete [11, [ND2 ] ].

An instance of the decision version of MAXLEAF is specified by supplying a graph $G$ and an integer $p$ and asking whether a spanning tree of $G$ exists which has at least $p$ leaves. Given an instance of MAXLEAF, we transform it into an instance of BCNS as follows. Set $W=\Delta+1$, where $\Delta$ is the maximum vertex degree in $G$. Obtain a proper $W$ edge coloring of $G$, which can be done in polynomial time as remarked above. Let $f(e)$ be the color assigned to edge $e$ by the proper coloring. For each edge $e$ of $G$, form the set $c(e)$ required for the BCNS instance as the singleton set containing $f(e)$. Finally, set $q=n-p$, where $n$ is the number of vertices in $G$.

Consider a vertex $v$ which is an interior node in the spanning tree in a solution to this instance of BCNS. By the construction, this vertex will have different colors on its incident edges in the tree, no matter what spanning tree is chosen, and hence will require a converter. Thus all interior vertices will require converters. On the other hand, a leaf vertex in the tree will not forward the broadcast to any other node and will not require a converter. Thus a solution to BCNS that uses no more than $q$ converters will use a tree with no more than $q$ interior nodes, that is a tree with no fewer than $n-q=p$ leaves. Hence if we could solve the
BCNS problem in polynomial time, we could also solve the MAXLEAF problem in polynomial time. Since MAXLEAF is known to be NP-Complete, so is BCNS.

It is significant to note that the same proof shows that BCNS with the restriction that $W \leq 5$ is still NP-complete, since MAXLEAF is NP-complete even for graphs with maximum vertex degree at most 4 . (However if we restrict to $W \leq 4$, the complexity of BCNS is left open by the above proof.)

2) Tractability of tree BCNS: In this section we present a recursive algorithm to obtain a solution to BCNS on trees.

a) Definitions:: Given a tree, $T$, arbitrarily choose a root, $r$ and root $T$ at $r$, directing all edges from parent to child.

- We use $p(v))$ to denote the parent of $v$ in (rooted) $T$.

- For node $v$ of $T$, let $T_{v}$ denote the subtree of $T$ rooted at $v$.

- Let children $(v)$ be the set of children of $v$ in $T$.

- Let $c c(v)$ be the set of colors common to every edge incident with $v, c c(v)=\cap_{e \in e_{T}(v)} c(e)$. (If $c c(v)=\emptyset$, then $v$ must have a converter.)

- Let $c_{p}(v)$ be the set of colors on the edge from vertex $v$ to its parent, $c_{p}(v)=c(\langle v, p(v)\rangle)$.

b) Objective:: Let $\operatorname{opt}(0, v)$ be the minimum number of converters required to route the traffic in $T_{v}$. We want opt $(0, r)$, where $r$ is the root of $T$. We introduce a slight generalization of the objective as follows: for $c$ a color, let opt $(c, v)$ be the minimum number of converters required to route the traffic in $T_{v}$, given that the traffic between $v$ and its parent in $T$ is carried on color $c$. The value of 0 for $c$ thus takes on the connotation of "not constrained a priori by color". This is consistent with our original notation since valid color values are in $\{1 \ldots W\}$.

We give a recursive definition of $\operatorname{opt}(0, r)$ and $\operatorname{opt}(c, v)$, then observe that it can be implemented with dynamic programming to run in time $O\left(n W^{2}\right)$.

c) The recursion:: We show the recursion for $\operatorname{opt}(0, v)$ and $\operatorname{opt}(c, v)$.

1) Base cases: For all $v$, for all $c$ such that $c>0$ and $c \notin c_{p}(v), o p t(c, v)=\infty$. Additionally, if $v$ is a leaf then for all $c \in c_{p}(v), o p t(c, v)=0$ and $\operatorname{opt}(0, v)=0$.

2) Recursive cases: We now consider those $\operatorname{opt}(c, v)$ such that $v$ is not a leaf and, if $c>0, c \in c_{p}(v)$.

We consider whether or not to put a converter at $v$. First observe that the minimum cost if $v$ is constrained to be a converter is

$$
A=1+\sum_{x \in \operatorname{children}(v)} \operatorname{opt}(0, x) .
$$

a) If $c>0$ and $c \notin c c(v)$, then $v$ must be a converter, so $\operatorname{opt}(c, v)=A$.

b) If $c=0$ and $c c(v)=\emptyset$, then $v$ must be a converter, so $\operatorname{opt}(0, v)=A$.

c) If $c>0$ and $c \in c c(v)$ :

If $v$ is not a converter, minimum number of converters is:

$$
B=\sum_{x \in \text { children }(v)} \operatorname{opt}(c, x) .
$$


If $c$ is a converter, minimum number of converters is $A$.

Take the minimum of these two:

$$
\operatorname{opt}(c, v)=\min (A, B) \text {. }
$$

d) If $c=0$ and $c c(v) \neq \emptyset$ :

If $v$ is a converter, minimum number of converters is $A$.

If $v$ is not a converter, any $c \in c c(v)$ may be used to carry all traffic on all edges in and out of $v$, and we need to pick the $c$ that gives the minimum number of converters. Find:

$$
D=\min _{c \in c c(v)}\left(\sum_{x \in \operatorname{children}(v)} \operatorname{opt}(c, x)\right) .
$$

Take the minimum of these two:

$$
\operatorname{opt}(0, v)=\min (A, D) \text {. }
$$

The above recursion can be proven correct by induction to show that the tree BCNS problem can be solved by this approach. It is also obvious that algorithm designed with such an approach will have polynomial running time. Below we briefly state such an algorithm.

d) Dynamic programming implementation:: Assume the nodes in the tree are ordered so that if $x$ is a child of $y$ then $x<y$, which can be easily done by, say, using reverse levelorder to list the vertices. Such a procedure is polynomial given any representation of the tree. We then fill in a $n \times(W+1)$ array opt $[1 . . n, 0 . . W]$ using the steps 1 and 2(a-d) above. This requires $\mathrm{O}\left(n W^{2}\right)$ time:

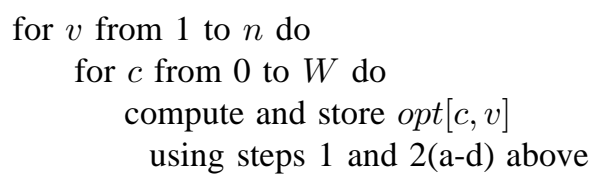

Note that when computing opt $[c, v]$, the values opt $\left[c^{\prime}, v^{\prime}\right]$ have already been computed and stored for all children $v^{\prime}$ of $v$ and all colors $c^{\prime}$, so any one needed can be looked up in constant time.

Finally, we point out that the procedure allows us to find not just the minimum number of converters, but the assignment of converters to vertices which gives the minimum. In addition to $o p t[c, v]$, we need to keep track of what choices led to the optimal value at each vertex. We do not include details of this simple procedure.

\section{Heuristic Algorithms}

In this section, we describe some heuristic approaches that we have developed for the general problem. Since we have an optimal algorithm for solving the BCNS problem on a tree network, we adopt the basic approach of first obtaining a spanning tree for the network, and then applying the tree algorithm. We consider the following flavors for generating the spanning tree.
- Random: In this heuristic, we simply remove randomly picked links from the network graph successively (unless such removal would disconnect the graph) until a spanning tree has been obtained. This approach is meant to serve as a baseline to compare the performance of the other algorithms.

- Color-richness: We define the color-richness of a link in the network as the total number of wavelengths available on that link. Intuitively, links which are more colorrich are better to retain in the tree, because the tree algorithm will have more options open. Accordingly, in this approach, we remove links in increasing order of color-richness, starting from the least color-rich one. Ties are broken arbitrarily.

- Weighted color-induced component score: Consider only a single wavelength $w$. If we consider only the links of the graph that have this wavelength available, and remove the rest, then in general the graph will become disconnected into several components. (If it does not, then this wavelength reaches all the nodes, and trivially this wavelength can be used to perform the broadcast without any converters.) We call these the components induced by the color $i$. Obviously, the nodes which are contained in a color-induced component of color $i$ can be reached from each other using color $i$ and no converters.

The color-richness measure in the previous heuristic can also be interpreted as the number of distinct color-induced components that a given links belongs in (because each free wavelength $w$ on a link implies the inclusion of that link in exactly one color-induced component of color $w)$. Intuitively, the larger the color-induced components, the less requirement for converters. Accordingly, in this heuristic, we compute a score for each link $l$ of the network as the sum of the sizes of the color-induced components link $l$ belongs to. That is, instead of taking a simple count of color-induced components as in the previous heuristic, in this one we weigh the count of color-induced components by the size of the colorinduced components. That is, we try to preserve larger color-induced components preferentially.

\section{NumericAl RESUlts}

To study the performance of our heuristic algorithms, we generated random network graphs with randomly assigned available wavelengths on the links. Densely connected graphs were generated by using a setting of "connectivity", a number between 0 and 1 , which is used as the probability of a link existing between any pair of nodes, independently. Thus the average degree in such graphs increases as the number of nodes in the graph. With a small number of nodes, such graphs often represent small network topologies well. Sparse graphs were generated by using a constant average degree for each node, where the nodes connected to any given node are picked randomly. Moreover the degree of each node in such graphs was held close to the average; that is the graphs are nearly regular. It is known that such graphs are often the simplest 


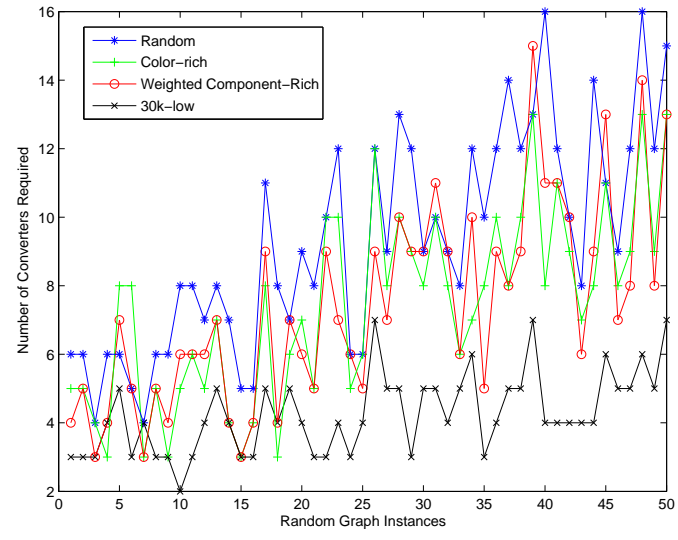

Fig. 3. Small dense networks, low connectivity

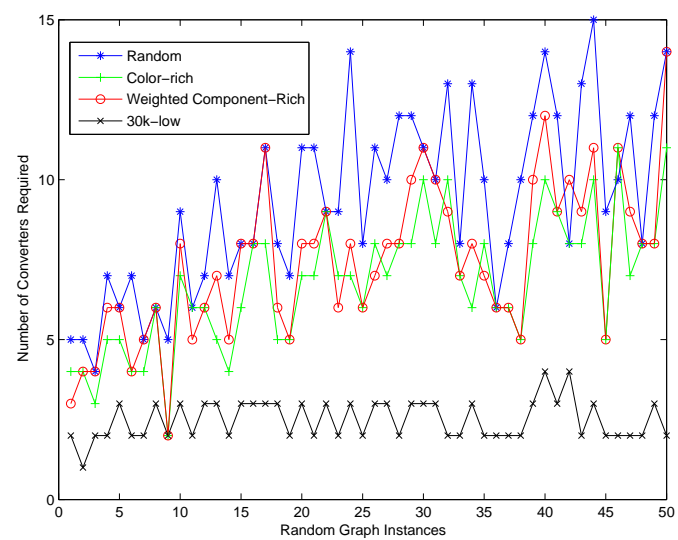

Fig. 4. Small dense networks, high connectivity

ones that adequately represent the computational complexity of intractable problems in practice. Also, such graphs are good candidates for network topologies when the number of nodes becomes large, for example it is well known that the average degree of nodes in the Internet is between 3 and 4 [8], [12]. The number of available wavelengths were generated by randomly choosing, with uniform distribution, a number between a specified minimum and maximum number of wavelengths. The actual wavelengths were again picked from the total number of wavelengths with uniform distribution.

We generated several families of problem instances, and between 40 and 50 instances for each family; here we present some representative results from these families. Families of "small" graphs were generated with number of nodes in the graph between 20 and 50, and the number of wavelengths between 32 and 80 . "Large" families were generated with number of nodes between 50 and 100, and number of wavelengths between 80 and 160 . The number of free wavelengths on each link were between 8 and 16 in both cases.

Figures 3-5 show results for different families of instances.

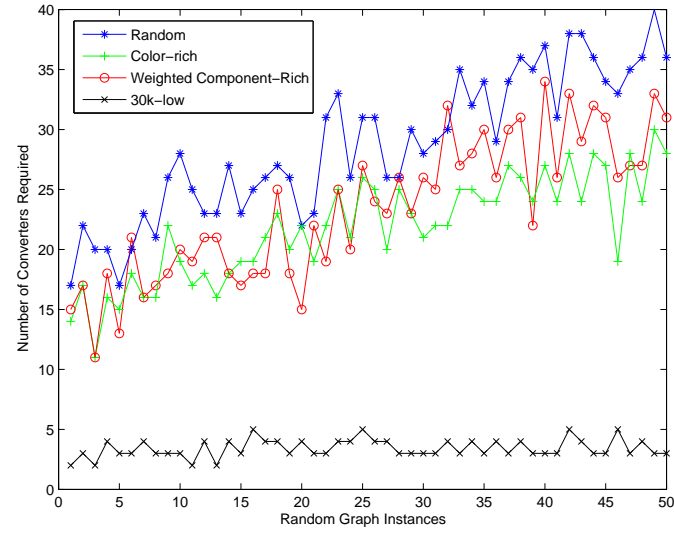

Fig. 5. Large dense networks, high connectivity

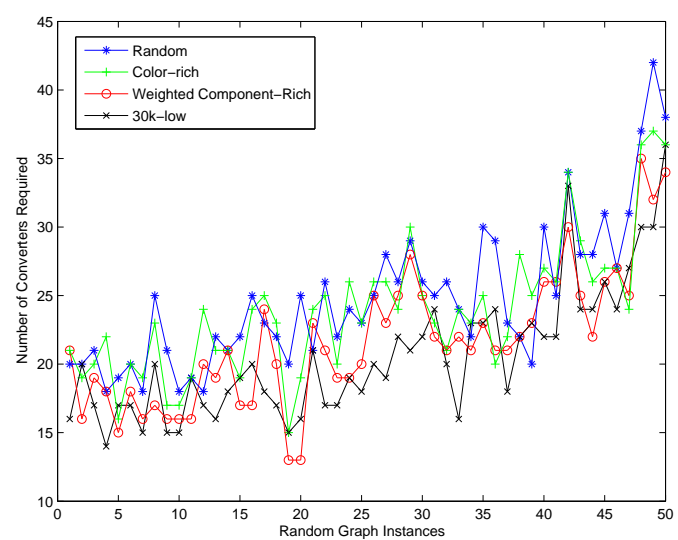

Fig. 6. Large sparse networks, average degree 3

Problem instances have been sorted by increasing number of nodes in the instance, for each family. As expected, the heuristics based on insight about the problem domain outperform the random heuristic in general. In certain cases, the random heuristic obtains a better result, but these cases are rare. Surprisingly, the heuristic based on color-induced components does not work significantly better than the colorrich heuristic as expected, but actually perform a little worse. This indicates that there may be a way to combine the strengths of the two approaches to obtain an even better solution. This is motivated further by latter results, see below.

To understand the absolute performance of our heuristics, it would be desirable to compare with the optimal results. However, finding the optimal is computationally prohibitive for the network sizes we consider. As a good indication of the optimal, we generated a large number $(30,000)$ of different spanning trees for each graph, and applied the tree algorithm to each, then adopted the best solution. For efficient generation of random spanning trees, we adopted an implementation by Donald Knuth of the algorithm given in [19]. Using this 


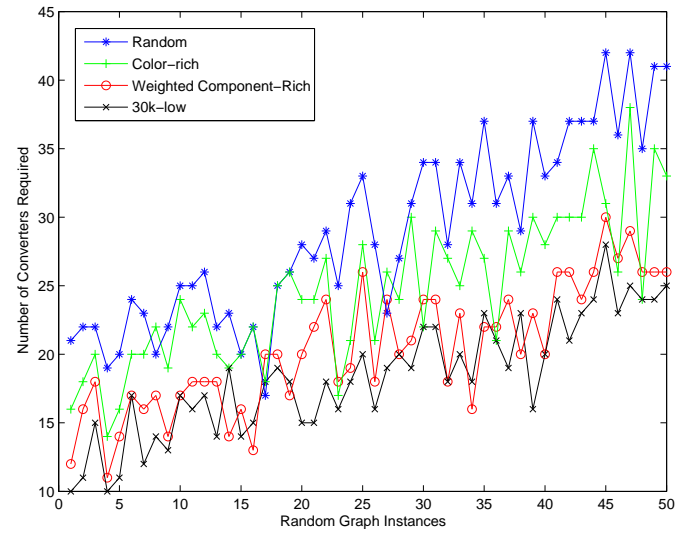

Fig. 7. Large sparse networks, average degree 8

algorithm, the 30,000 trees are generated in a matter of minutes for even the large family of graphs, hence this approach may count as a practical heuristic method in itself, even though it is more computation intensive than the heuristic approaches we propose. The best result from the 30,000 trees is plotted for each of the graphs as well, with the legend "30-k low". As we can see, our heuristic approaches are not very far from the 30-k low for the small family of graphs; in other words the heuristic approaches are good in an absolute sense for small dense graphs. However, for large dense graphs, the results of our heuristic approaches, though still better than the random approach, are significantly far from the 30-k low. We have conjectured that this is due to the unrealistic nodal degrees of the large dense family of graphs (in a 100-node network with connectivity 0.7 , the average degree of a node is 70 ). Thus some very shallow spanning trees may exist, with a large number of leaves, and the number of converters optimally required may be low for this reason.

To verify this intuition, we experimented with large sparse graphs of nearly regular degree as described above. As we can see from Figures 6-7, experimental results validate our conjecture. The heuristic approaches are in the same range as the 30-k low in these sets. In the sparse family with average degree 3 , all the approaches have performances close to each other, probably because good solutions are rare and the optimal is hard to find even for the 30-k search. For the family with average degree 8 , the $30-\mathrm{k}$ low is clearly better than the random, and also the color-rich heuristic. However, the weighted color-induced component heuristic is as good as the 30-k low, showing that it is good in an absolute sense. This also further motivates our earlier observation that neither of the two heuristics is always better than the other, and combining their strengths is a worthwhile topic for future research.

In the above, we have not attempted to compare our experimental results with those in [16], [17], because there is no meaningful way to make such a comparison. Examples can be constructed where a solution to the BCNS problem requires $(n-1) / 3$ converters, but the bounded approximation algorithms in [16], [17] would return only $O(\ln n)$ converters. The problem is that those algorithms are solving the CCVS problem. It is not even clear that it can be checked in polynomial time whether a solution to CCVS is a solution to BCNS or not.

\section{CONCLUSion}

We have considered the problem of the placement of converter nodes in a WDM network in support of broadcast. Prior work on this problem exists, but we have shown that the prior work erroneously focuses on a related but different problem, so the solutions obtained by the algorithms in these studies may fail to find feasible solutions to the BCNS problem. Moreover, the question of inherent complexity of this problem has been left open.

By formulating the problem as a coloring problem, we were able to show that the problem is NP-Complete in general. However, for tree networks an optimal solution may be obtained in polynomial time; we showed this by recursive arguments and also showed how it may be computed by a dynamic programming algorithm of low complexity. For general networks, we proposed heuristic approaches that appear to have good practical performances. In our ongoing work, we are investigating the possibility of combining such approaches utilizing domain specific insights with standard algorithms for problems such as MAXLEAF in order to obtain heuristics that are approximation algorithms for this NP-Complete problem.

\section{ACKNOWLEDGEMENTS}

We would like to acknowledge some useful discussions with Abhishek Joshi on the issues addressed in this paper.

\section{REFERENCES}

[1] P.L. Belotti and T.J.K. Stidsen. Optimal placement of wavelength converting nodes. DRCN 2001. Third International Workshop on Design of Reliable Communication Networks. 'Price and Value of Reliability'. Proceedings, pages $15-21,2001$.

[2] Tak-Ming Chan, S. Kwong, and K.F. Man. Solving the converter placement problem in wdm ring networks using genetic algorithms. Computer Journal, 46(4):427 - 48, 2003.

[3] Guangting Chen, Guojun Li, and Guoliang Xue. Optimal placement of wavelength converters in wdm optical networks with a general tree of rings topology. Proceedings Ninth International Conference on Computer Communications and Networks (Cat.No.00EX440), pages 606 $-11,2000$.

[4] Xiaowen Chu, Bo Li, and I. Chlamtac. Wavelength converter placement under different rwa algorithms in wavelength-routed all-optical networks. IEEE Transactions on Communications, 51(4):607 - 17, 2003.

[5] Xiaowen Chu, Bo Li, Jiangchuan Liu, and Lizhong Li. Wavelength converter placement under a dynamic rwa algorithm in wavelengthrouted all-optical networks. 2002 International Conference on Communications, Circuits and Systems and West Sino Exposition Proceedings (Cat. No.02EX591), vol.1:865 - 70, 2002.

[6] R. Dutta and G.N. Rouskas. A survey of virtual topology design algorithms for wavelength routed optical networks. Optical Networks Magazine, 1(1):73-89, Jan 2000.

[7] R. Dutta and G.N. Rouskas. Traffic grooming in WDM networks: Past and future. IEEE Network, 16(6):46-56, Nov/Dec 2002

[8] M. Faloutsos, P. Faloutsos, and C. Faloutsos. On power-law relationships of the internet topology. Comput.Commun. Rev., pages 251-262, 1999.

[9] Y.C. Foo, Youngseok Lee, C.F. Teo, S.F. Chien, and A.L.Y. Low. Enhancing wavelength converter placement optimization with trafficengineering-aware shortest-path routing. IEICE Electronics Express, 1(14), 2004. 
[10] Suixiang Gao, Xiaohua Jia, Chuanhe Huang, and Ding-Zhu Du. An optimization model for placement of wavelength converters to minimize blocking probability in wdm networks. Journal of Lightwave Technology, 21(3):684 - 94, 2003.

[11] M.R. Garey and D.S. Johnson. Computers and Intractability: A Guide to the Theory of NP-Completeness. W.H.Freeman and Company.

[12] R. Govindan and H. Tangmunarunkit. Heuristics for internet map discovery. In Proc. 19th IEEE Conf. on Computer Communications (INFOCOM), pages 1371-1380, 2000.

[13] Quang-Dzung Ho, Nga Dinh-Thi-Thuy, Minh-Ho Rang, and ManSeop Lee. A heuristic converter placement scheme for wavelengthrouted optical networks. 6th International Conference on Advanced Communication Technology (IEEE Cat. No.04EX801), Vol.2:607 - 10, 2004.

[14] Xiao-Hua Jia, Ding-Zhu Du, Xiao-Dong Hu, He-Jiao Huang, and DeYing Li. On the optimal placement of wavelength converters in wdm networks. Computer Communications, 26(9):986 - 95, 2003.

[15] S. Kundu and T. Yi. Optimal converter-placement in tree networks. Proceedings of the ISCA 15th International Conference Parallel and Distributed Computing Systems, pages 337 - 42, 2002.

[16] Lu Ruan, Dingzhu Du, Xiaodong Hu, Xiaohua Jia, Deying Li, and Zheng Sun. Converter placement supporting broadcast in wdm optical networks. IEEE Transactions on Computers, 50(7):750 - 8, 2001.

[17] Lu Ruan and Weili Wu. Broadcast routing with minimum wavelength conversion in wdm optical networks. Journal of Combinatorial Optimization, 9:223-235, 2005.

[18] J.H. Siregar, H. Takagit, and Y. Zhang. Optimal wavelength converter placement in optical networks by genetic algorithm. IEICE Transactions on Communications, E85-B(6):1075 - 82, 2002.

[19] M.J. Smith. Generating spanning trees, 1999.

[20] S. Subramaniam, M. Azizoglu, and A.K. Somani. On optimal converter placement in wavelength-routed networks. IEEE/ACM Transactions on Networking, 7(5):754 - 66, 1999.
[21] V. Tamarapalli and S.H. Srinivasan. Wavelength converter placement in wdm networks with non-uniform traffic. 13th IEEE Workshop on Local and Metropolitan Area Networks (IEEE Cat No.04EX873), pages 109 12, 2004.

[22] Wu Tao, Sun Junqiang, Liu Deming, and Huang Dexiu. The optimal placement of sparse wavelength converters in wdm networks based on genetic algorithms. Journal of Huazhong University of Science and Technology, 29(8):10 - 11, 2001.

[23] S. Thiagarajan and A.K. Somani. Optimal wavelength converter placement in arbitrary topology wavelength-routed networks. Computer Communications, 26(9):975 - 85, 2003.

[24] K.R. Venugopal, M. Shivakumar, and P.S. Kumar. A heuristic for placement of limited range wavelength converters in all-optical networks. IEEE INFOCOM '99. Conference on Computer Communications. Proceedings. Eighteenth Annual Joint Conference of the IEEE Computer and Communications Societies. The Future is Now (Cat. No.99CH36320), vol.2:908 - 15, 1999.

[25] K.R. Venugopal, M. ShivaKumar, and P.S. Kumar. A heuristic for placement of limited range wavelength converters in all-optical networks. Computer Networks, 35(2-3):143 - 63, 2001.

[26] C. Vijayanand, M.S. Kumar, K.R. Venugopal, and P.S. Kumar. Converter placement in all-optical networks using genetic algorithms. Computer Communications, 23(13):1223 - 34, 2000.

[27] Peng-Jun Wan, O. Frieder, and Liwu Liu. Optimal placement of wavelength converters in trees, tree-connected rings, and tree of rings. Computer Communications, 26(7):718 - 22, 2003.

[28] Ye Wang, Lemin Li, and Sheng Wang. A new algorithm of design protection for wavelength-routed networks and efficient wavelength converter placement. ICC 2001. IEEE International Conference on Communications. Conference Record (Cat. No.01CH37240), vol.6:1807 $-11,2001$.

[29] D.B West. Introduction to Graph Theory. Prentice Hall, 1996. 